\title{
LC3A, LC3B and Beclin-1 Expression in Gastric Cancer
}

\author{
ALEXANDRA GIATROMANOLAKI ${ }^{1}$, MICHAEL I. KOUKOURAKIS $^{2}$, \\ IOANNIS GEORGIOU ${ }^{1}$, MARIA KOUROUPI ${ }^{1}$ and EFTHIMIOS SIVRIDIS ${ }^{1}$ \\ Departments of ${ }^{1}$ Pathology, and ${ }^{2}$ Radiotherapy/Oncology, \\ Democritus University of Thrace and University General Hospital of Alexandroupolis, Alexandroupolis, Greece
}

\begin{abstract}
Background: The current study examined the key proteins involved in autophagosome formation and their prognostic role in gastric cancer. Materials and Methods: Paraffin-embedded tissues from 121 consecutive patients treated with surgery for gastric cancer were analyzed immunohistochemically for the expression of autophagic proteins microtubule-associated proteins $1 \mathrm{~A} / 1 \mathrm{~B}$ light chain $3 \mathrm{~A}$ and $3 B(L C 3 A, L C 3 B)$ and beclin-1 (encoded by BECN1 gene). Assessment of proliferative index using E3 ubiquitin-protein ligase (MIB1) and correlations with histopathological parameters and overall survival were performed. Results: Strong cytoplasmic expression was noted for all studied proteins, although to a varying proportion, the median percentage being $30 \%$ for $L C 3 A$, and $40 \%$ for $L C 3 B$ and beclin-1. The median score of $\mathrm{LC} \mathrm{A}^{+}$stone-like structures (SLS) was 0.2 (range 0-1) and the median proliferative index was $30 \%$ (range $=0-95 \%$ ). A significant association between $L C 3 A, L C 3 B$ and beclin-1 expression was confirmed ( $p<0.01)$. SLS score was higher in tumors of the gastro-esophageal junction ( $p=0.009)$, and beclin-1 was overexpressed in intestinal-type tumors $(p=0.001)$. High SLS score $(p=0.008)$ was significantly related to poor prognosis, and this finding persisted in multivariate analysis (hazard ratio $(H R)=2.01$, $p=0.003)$. Conclusion: Intense autophagic activity, as assessed by LC3A immunostaining and SLS quantification, is a strong prognostic marker in gastric cancer and can be useful for clinical application.
\end{abstract}

The role of autophagy in cancer development, growth and metastasis has been recently under intense investigation. The overexpression of proteins involved in the autophagic pathway has emerged as being of prognostic relevance in

Correspondence to: Alexandra Giatromanolaki, Democritus University of Thrace, Alexandroupolis 68100 , Greece. Tel/Fax: +30 255175117, e-mail: targ@her.forthnet.gr; agiatrom@med.duth.gr

Key Words: Gastric cancer, autophagy, LC3A, LC3B, beclin 1, stone-like structure, SLS. several cancer types, including gastrointestinal malignancies (1-3). Microtubule-associated proteins 1A/1B light chain 3A and $3 \mathrm{~B}$ (LC3A and $\mathrm{LC} 3 \mathrm{~B}$ ) proteins are among the most studied, as they constitute the essential proteins of the inner and outer autophagosomal membranes, providing the means of an indirect estimate of the cellular autophagosomal content.

Three main isoforms of the LC3 protein, encoded by different genes, have been identified, namely LC3A, -B and $-\mathrm{C}(4,5)$. These may not be identical in their function and, as we recently reported, the three LC3 isoforms possess distinct subcellular localizations and nuclear/cytoplasmic kinetics (6). Moreover, aside from the overexpression of these proteins by a subset of carcinomas, an LC3A-specific expression pattern, the so called 'stone-like structure' (SLS) pattern identified by our group, has shown the strongest association with prognosis in a variety of human tumor types (7-9). The SLS pattern is exclusively found in cancer cells and is recognized as large, rounded, densely stained material, amorphous or laminated, typically enclosed within an LC3Aexpressing cytoplasmic vacuole (10). We have postulated that this pattern of expression goes along with an intense autophagic flux that exhausts the lysosomal pool necessary for the digestion of the autophagosomal content, resulting in the accumulation of undigested material in the cytoplasm of cells. Of interest, glucose deprivation and acidic conditions reproduce this pattern of expression in cell culture (11).

In the current study, we investigated the expression patterns of the autophagic proteins LC 3A, LC3B and that of the autophagy initiation signaling molecule beclin-1 in a series of gastric carcinomas treated with surgery. The role of these proteins in the prognosis of patients was also analyzed.

\section{Materials and Methods}

Formalin-fixed, paraffin embedded material from 121 patients treated with curative surgery for gastric cancer were collected from the Archives of the Department of Pathology, Democritus University of Thrace, Medical School, Alexandroupolis, Greece. The selected cases were serial according to the archive number. Table I reports the patient and disease characteristics. The median overall survival was 38 months 
(range=2-156 months) and 41/121 patients were alive at the time of analysis. Patients were classified according to the pathologic TNM International Union Against Cancer staging system (12).

Immunohistochemistry for autophagy/lysosome related proteins. Autophagy-related proteins were detected by a previously standardized immunohistochemical technique. Selection of the LC3A and LC3B antibodies was performed based on a previous study of validation of the specificity of multiple antibodies regarding the selective recognition of two out of three known LC3 isoforms (6). The primary antibodies used were as follows: i) Purified rabbit polyclonal antibody MAP1LC3A (Abgent, San Diego, CA, USA) raised against a synthetic peptide at the $\mathrm{C}$-terminal cleavage site of the human cleaved-LC3A (APG8a). ii) Mouse monoclonal MAP1LC3B 5F10 (Nanotools, Teningen, Germany) antibody used at a dilution 1:100 with overnight incubation. iii) Rabbit monoclonal antibody EPR1733Y to beclin-1 (Abcam, Cambridge, UK), used at a dilution 1:50 with overnight incubation. This was raised against a synthetic peptide corresponding to residues near the C-terminus of human beclin-1. For the assessment of the proliferative index, MIB1 monoclonal antibody (DAKO, Glostrup, Denmark) was used at a dilution of 1:75 for $20 \mathrm{~min}$.

Sections, 3- $\mu \mathrm{m}$-thick, were deparaffinized and placed in antigen retrieval target solution $\mathrm{pH} 9.0$ (DAKO), followed by microwaving $(3 \times 5 \mathrm{~min})$. The primary monoclonal antibodies were applied. Following washing with phosphate-buffered saline (PBS), endogenous peroxidase was quenched with EnVision Flex Peroxidase Block (DAKO) for 10 min then sections were washed with PBS. Non-specific binding was blocked in EnVision Flex mouse Linker for 15 min (DAKO), and then sections were washed with PBS. Sections were then incubated with a secondary antibody (EnVision Flex/HRP; DAKO) for $30 \mathrm{~min}$, and washed in PBS. The color was developed by 5 min incubation with 3,3'-diaminobenzidine (DAB) solution and sections were counterstained weakly with hematoxylin.

In every staining run, one component of the sequential staining reaction was omitted from at least one section, this usually being the omission of the primary antibody and its replacement by normal rabbit immunoglobulin-G.

Evaluation of immnunohistochemistry. The patterns of LC3A expression are mainly diffuse cytoplasmic and SLS, as previously reported (7-9). The SLS are readily recognized as large, rounded, densely stained material, amorphous or laminated, typically enclosed within a $\mathrm{LC}^{+}$cytoplasmic vacuole. The proportion of cancer cells with strong $\mathrm{LC} \mathrm{A}^{+}$cytoplasmic expression was recorded in all available $\times 200$ optical fields (whole section) and the mean value was used to score each case. For SLS scoring, the number of cells with SLS features was recorded in all available $\times 200$ optical fields (whole section) and the score was obtained by dividing the total number of SLS by the number of optical fields. LC3B has a predominantly cytoplasmic staining pattern The proportion of cancer cells with strong LC3B cytoplasmic expression was recorded in all available $\times 200$ optical fields (whole section) and the mean value was used to score each case.

MIB1 expression is nuclear and therefore the percentage of cancer cells with nuclear immunostaining was assessed in all available fields ( $\times 200$ magnification $)$ and the mean value was used to score each case.

For all markers, values higher than the median were used to group cases as having high or low protein expression.
Table I. Patient and disease characteristics.

\begin{tabular}{ll}
\hline Parameter & Value \\
\hline Gender, $\mathrm{n}$ & \\
Female & 36 \\
Male & 85 \\
Age, $\mathrm{n}$ & \\
39-65 Years & 45 \\
>65 Years & 76 \\
Status, $\mathrm{n}^{*}$ & \\
Alive & 41 \\
Dead & 80 \\
Follow-up (months) & \\
Median (range) & $38(2-156)$ \\
Tumor localization, $\mathrm{n}$ & \\
Body & 70 \\
Pylorus & 42 \\
Gastro/esophageal junction & 9 \\
Histology, $\mathrm{n}$ & \\
Diffuse & 25 \\
Intestinal & 96 \\
T-Stage, $\mathrm{n}$ & \\
1,2 & 43 \\
3 & 78 \\
Nodal status, n & \\
Negative & 51 \\
Positive & 70 \\
Grade (intestinal-type), $\mathrm{n}$ & \\
1 & 26 \\
2 & 19 \\
3 & 51 \\
\hline
\end{tabular}

*Last follow-up recorded in patient documents or retrieved by contacting the patient or their relatives.

Statistical analysis. The statistical analysis and graphical presentation was performed using GraphPad Prism 5.00 and the GraphPad Instat packages (GraphPad, San Diego, CA, USA). The chi-squared or the Fisher's exact test was used to compare categorical variables, as appropriate. Linear regression analysis was used to assess correlations between continuous variables. Survival curves were plotted using the Kaplan-Meier method, and the logrank test was used to determine statistical differences between life tables. A Cox proportional hazard model was used to assess the effect of assessed parameters on death events. $p$-Values less than 0.05 were considered to be statistically significant.

\section{Results}

Histopathological correlations. Analysis of associations between histopathological variables revealed a strong association between T-stage and nodal involvement $(p<0.0001)$. In the group of intestinal-type adenocarcinomas, high grade was directly related to advanced T-stage $(p=0.02)$ and nodal involvement $(p=0.0006)$. The proliferative index ranged from $0-95 \%$ (median $=30 \%$ ). No association of LC3 or beclin-1 proteins with histopathological variables was noted. 

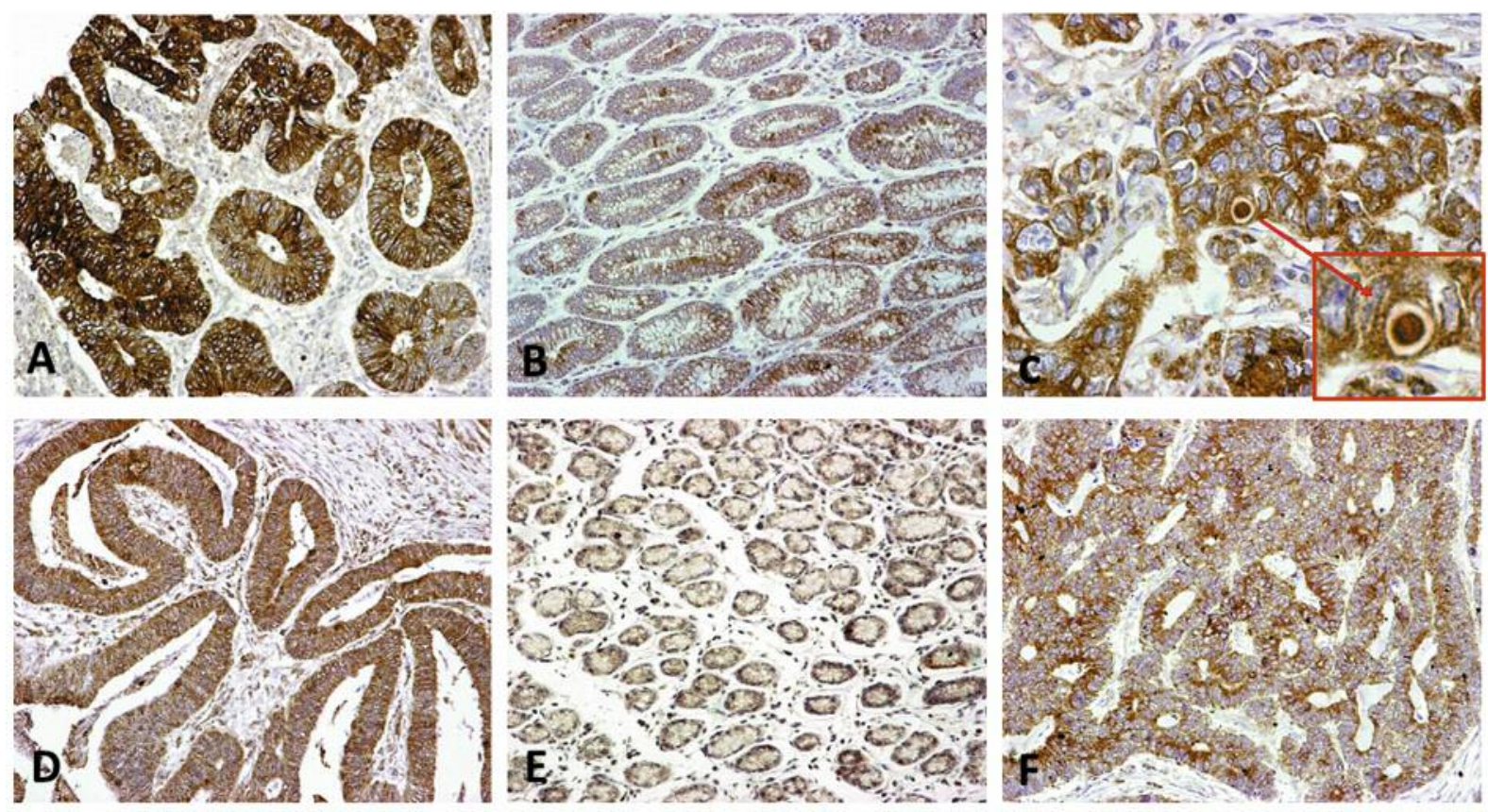

Figure 1. Immunohistochemical images of gastric cancer and normal gastric mucosa. A: Strong cytoplasmic expression of microtubule-associated proteins $1 A / 1 B$ light chain $3 A(L C 3 A)$ protein by cancer cells in a gastric adenocarcinoma $(\times 20)$. B: Weak cytoplasmic expression of LC3A by normal gastric glands $(\times 20)$. C: A typical LC3A-positive stone-like structure $(\times 20$; inset: $\times 60)$. D: Strong cytoplasmic expression of LC3B in a gastric adenocarcinoma $(\times 20)$. E: Weak cytoplasmic expression of LC3B by normal gastric glands $(\times 20)$. F: Strong cytoplasmic beclin-1 expression in a gastric adenocarcinoma $(\times 20)$.

Correlations between immunohistochemical variables. Strong cytoplasmic expression of LC3A was noted to a varying degree among cases, the median percentage being $30 \%$ (range $=0-100 \%)$. Cases with $\mathrm{LC} \mathrm{A}^{+} \geq 30 \%(74 / 121$, $61.2 \%)$ were considered as having high cytoplasmic LC3A expression. Figure 1A shows a typical image of strong LC3A cytoplasmic staining in cancer cells, while Figure 1B shows weak expression in normal gastric mucosa. The median score for LC3A+ SLS was 0.2 (range=0-1). Sixty-four out of 121 $(52.9 \%)$ cases had a score $\geq 0.2$ and these cases were considered as having a high SLS score. Figure 1C shows a typical SLS among cancer cells.

Regarding LC3B, the median percentage of cells with strong reactivity was $40 \%$ (range $=0-100 \%$ ). Sixty-three out of $121(52 \%)$ cases expressed LC $3 B$ in $\geq 40 \%$ of the cell population, and these were assigned to the category with high LC3B expression. Figure 1D shows a typical image of strong LC3B cytoplasmic staining in cancer cells, while Figure 1E shows weak expression in normal gastric mucosa.

Beclin-1 was strongly expressed in a median of $40 \%$ of cancer cells (range 0-100\%), and 62/121 (51.2\%) that had a score $\geq 40 \%$ were considered to have high beclin- 1 reactivity. Figure $1 \mathrm{~F}$ shows typical expression of beclin-1 by gastric cancer cells.
Linear regression analysis between the immunohistochemical variables studied revealed a strong positive association between cytoplasmic LC3A expression and the SLS score $(p<0.0001$, $\mathrm{r}=0.26)$ as well as with LC3B expression $(p=0.002, \mathrm{r}=0.26)$. LC3B expression was also directly positively associated with beclin-1 expression $(p<0.0001, \mathrm{r}=0.46)$. Although there was a trend, the correlation of SLS and LC3A with beclin-1 expression did not reach significance.

Categorical analysis revealed similar associations (Table II). Although overall there was a significant concurrent overexpression of LC3A and LC3B in gastric carcinomas ( $p=0.002), 30 / 121$ cases had preferentially up-regulated expression of LC3A, while 14/121 overexpressed only the LC3B protein. In categorical analysis, a significant positive association of LC3A and of LC3B with beclin-1 expression was confirmed ( $p=0.009$ and $p=0.0001$, respectively).

Association between immunohistochemical and histopathological variables. The only significant association between LC3A or SLS and histopathological variables was the significantly higher SLS score noted in tumors of the gastroesophageal junction as compared to those of the gastric body or pyloric regions (medial SLS score: $0.45 v s .0 .23$ and 0.20 respectively; $p=0.009$ ). By contrast, tumors of junctional 
Table II. Analysis of the association between immunohistochemical variables by category.

\begin{tabular}{|c|c|c|c|c|c|c|c|c|c|}
\hline & \multicolumn{2}{|c|}{ LC3A, n } & \multirow[b]{2}{*}{$p$-Value } & \multicolumn{2}{|c|}{ LC3B, n } & \multirow[b]{2}{*}{$p$-Value } & \multicolumn{2}{|c|}{ SLS, n } & \multirow[b]{2}{*}{$p$-Value } \\
\hline & Low & High & & Low & High & & Low & High & \\
\hline LC3B & 47 & 74 & & 58 & 63 & & 57 & 64 & \\
\hline Low & 31 & 27 & 0.002 & & & & & & \\
\hline High & 16 & 47 & & & & & & & \\
\hline \multicolumn{10}{|l|}{ SLS } \\
\hline Low & 36 & 21 & $<0.0001$ & 29 & 28 & 0.58 & & & \\
\hline High & 11 & 53 & & 29 & 35 & & & & \\
\hline \multicolumn{10}{|l|}{ Beclin-1 } \\
\hline Low & 30 & 29 & 0.009 & 39 & 20 & 0.0001 & 33 & 26 & 0.06 \\
\hline High & 17 & 45 & & 19 & 43 & & 24 & 38 & \\
\hline
\end{tabular}

LC3A, LC3B: Microtubule-associated proteins 1A/1B light chain 3A and 3B proteins; SLS: stone-like structure.

location had a significantly lower expression of LC3B $(21 \%$ vs. $43 \%$ and $39 \%$ for body and pyloric locations, respectively; $p=0.05)$. Beclin-1 was significantly overexpressed in intestinaltype adenocarcinomas compared to diffuse-type tumors (median 70\% vs. 35\%; $p=0.001$ ). No other association of any of the immunohistochemical variables examined with $\mathrm{T}$ - or $\mathrm{N}$ stage was noted.

Survival analysis. Kaplan-Meier disease-specific overall survival analysis showed that advanced T-stage of disease, nodal involvement, diffuse growth pattern and high grade in intestinal-type tumors were significantly related to poor prognosis (Table III). A high SLS score was significantly related to poor prognosis $(p=0.008)$ and this persisted in multivariate analysis $(p=0.003$, hazard ratio=2.01); Figure 2 . High expression of LC3B was related to a favorable outcome in univariate analysis, although this did not reach significance $(p=0.08)$.

\section{Discussion}

Autophagy is an essential biological process aiming to cleanse the cell cytoplasm of damaged or aged proteins and organelles which are used in the body's innate recycling program for producing simple molecular structures for energy and growth demands (13). In this process, the degraded material is engulfed by autophagosomes and subsequently fused with lysosomes, the enzymes responsible for digestion of the intra auto-lysosomal material. Up-regulation of autophagy flux often occurs in cancer cells, providing them with an important survival and growth advantage (14).

Beclin-1, a mammalian orthologue of the yeast apg6/vps30 gene, is a scaffold protein for the formation of the phosphatidylinositol-3-kinase (PI3K) complex (15). This protein complex is among the first components recruited by
Table III. Univariate (Kaplan-Meier) and Cox regression multivariate analysis of death events.

\begin{tabular}{|c|c|c|c|c|}
\hline \multirow[b]{2}{*}{ Variable } & \multicolumn{2}{|c|}{ Univariate } & \multicolumn{2}{|c|}{ Multivariate } \\
\hline & HR & $p$-Value & HR & $p$-Value \\
\hline \multicolumn{5}{|l|}{ Histology } \\
\hline Diffuse $v s$. intestinal & 1.97 & 0.02 & ---- & ---- \\
\hline \multicolumn{5}{|l|}{ Location } \\
\hline Pylorus vs. other & 3.90 & 0.01 & 2.93 & 0.005 \\
\hline \multicolumn{4}{|l|}{ T-Stage } & 0.01 \\
\hline \multicolumn{5}{|l|}{ Node } \\
\hline Positive $v s$. negative & 2.17 & 0.0009 & 1.92 & 0.01 \\
\hline $\begin{array}{l}\text { Grade intestinal type } \\
\quad 3 \text { vs. } 1 / 2\end{array}$ & 1.90 & 0.01 & --- & ---- \\
\hline \multicolumn{5}{|l|}{ Proliferative index } \\
\hline $\begin{array}{l}\text { High vs. low } \\
\text { LC3A }\end{array}$ & 0.80 & 0.30 & LC3A & ---- \\
\hline High $v s$. low & 0.78 & 0.29 & ---- & ---- \\
\hline $\begin{array}{l}\text { LC3A SLS } \\
\text { High vs. low }\end{array}$ & 1.81 & 0.008 & 2.01 & 0.003 \\
\hline \multicolumn{5}{|l|}{ LC3B } \\
\hline $\begin{array}{l}\text { High } v s . \text { low } \\
\text { Beclin-1 }\end{array}$ & 0.59 & 0.08 & ---- & --- \\
\hline High $v s$. low & 1.01 & 0.98 & ---- & ---- \\
\hline
\end{tabular}

HR: Hazard ratio; LC3A, LC3B: microtubule-associated proteins 1A/1B light chain $3 \mathrm{~A}$ and $3 \mathrm{~B}$ proteins; SLS: stone-like structure.

the developing autophagosome and is essential for autophagy. The clinicopathological role of PI3K protein in gastric cancer has been extensively studied, but yielded contradictory results in regard to its prognostic value. Studies from Chinese institutions reported that the immunohistochemical overexpression of gastric carcinomas ranges between $58 \%$ and $84 \%$ (16-19). It was found that reduced beclin-1 

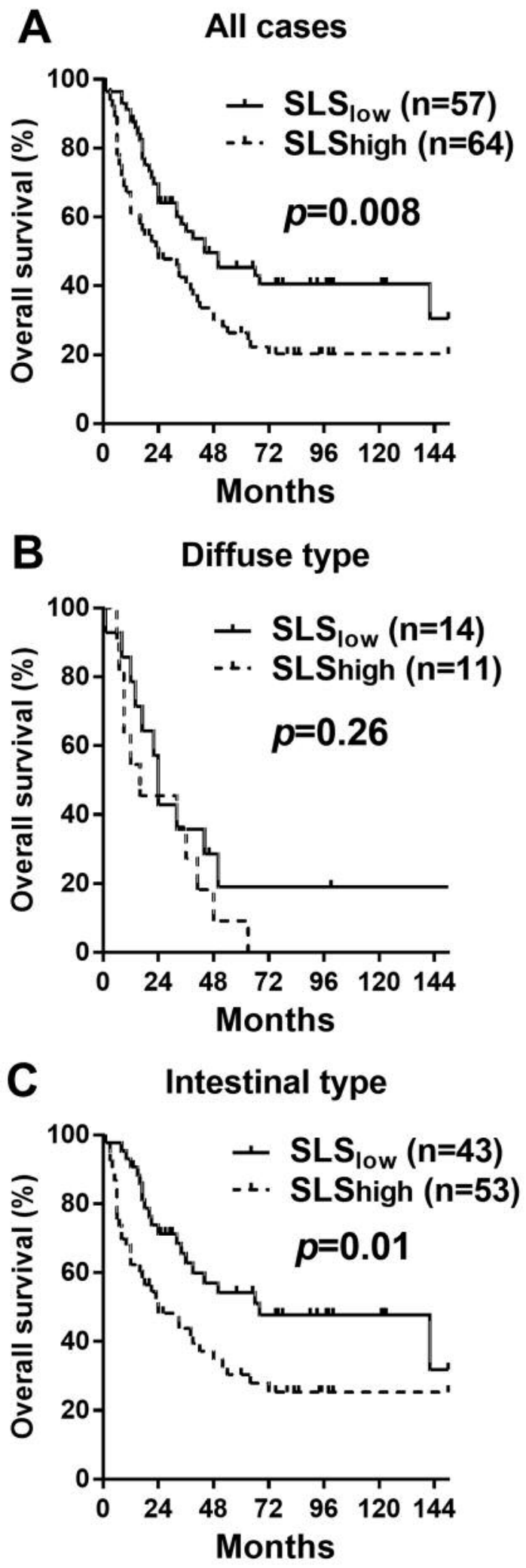

Figure 2. Kaplan-Meier disease-specific overall survival curves of all patients $(A)$, patients with diffuse-type $(B)$ and those with intestinal-type (C) gastric cancer. expression was associated with poor differentiation, advanced stage and shorter overall survival. In 2015, a study form Korea reported similar results, with the reduced beclin-1 expression being related to high grade and advanced-stage tumors (20). A more recent Chinese study reported that beclin-1 overexpression was linked with poor prognosis in their series of patients (21).

In our study, beclin-1 overexpression was found in 51\% of cancer cases and this was associated with the intestinal-type gastric adenocarcinomas - a finding which is in accordance with the study by Yu et al. (19). We were not able, however, to confirm any positive or negative prognostic relevance with regard to beclin-1. Whether ethnicity is a factor defining the distinct biological function of beclin-1 between Western and Eastern populations, which may differently affect prognosis, cannot be excluded as a reason for this discrepancy $(22,23)$. Suppression of beclin-1 may occur for various reasons and this may characterize a subgroup of patients with poor prognosis. This, however, does not mean that such tumors do not use alternative pathways to activate autophagy. The role of beclin-1 can be well bypassed in its absence, so that high autophagy flux may exist even in beclin-1-negative tumors $(24,25)$. Thus, beclin- 1 is not a reliable marker of autophagy flux. Aside from its role in autophagy, it has additional functions that may affect cancer biological behavior independently of autophagy (26)

We, therefore, focused on structural autophagosomal proteins in order to assess the autophagosomal content in cancer cells, using immunohistochemistry. It is stressed that even such markers can fail to estimate the autophagic flux, as accumulation of autophagosomes can be either an indicator of reduced autophagosomal consumption or of increased autophagosomal formation. In any case, the LC3 proteins seem to be the most appropriate markers for studying the autophagosomal cytoplasmic content, as these are essential components of the inner and outer autophagosomal membranes (13). We examined the expression of two distinct forms of the LC3 protein, LC3A and LC3B. We found a significant association between the cytoplasmic expression of the two forms, although in onethird (44/121) of the examined cases expression of only one of these two LC3s was up-regulated. Expression of both proteins was significantly positively correlated with that of beclin-1, showing that beclin-1 was an important autophagy signaling molecule in a large percentage of the gastric carcinomas examined. No association with histopathological variables or MIB1 proliferative index was noted.

An additional LCA-related immunohistochemical pattern was studied - the so-called SLS, which, presumably, reflects extensive cytoplasmic consumption of the cancer cell and exhaustion of the lysosomal content that can be readily recognized and quantified in cancer specimens, as previously reported (8-10). This pattern was directly linked with 
cytoplasmic LC3A expression and was the only autophagyrelated parameter that was strongly and independently linked with poor prognosis. This is in accordance with a recently published study on gastric cancer (27). Cytoplasmic expression of LC3A was not related to prognosis, while overexpression of LC3B was marginally associated with better outcome, compatible with the results reported in an earlier study (28). Cytoplasmic expression of LC3A was previously found to be associated with good prognostic markers in breast cancer (9). It seems that the cytoplasmic expression of LC3 proteins, although identifying tumors of high autophagic activity, also identifies tumors with a reduced autophagic flux resulting in autophagosome accumulation. The LC $3 \mathrm{~A}^{+}$SLS pattern is a finding that can discriminate which tumors have intense autophagic flux, and these tumors, indeed, had an aggressive clinical behavior (8-10).

It is concluded that intense autophagic activity, as assessed with LC3A immunostaining and SLS pattern quantification, is a strong prognostic marker in gastric cancer and can be tested for clinical use, aside from the traditional histopathological prognostic features.

\section{Ethical Standards}

The study was approved by the local University Ethics and Research Committee (protocol number: ES 3/7-4-2011).

\section{Conflicts of Interest}

The Authors declare that they have no conflict of interest in regard to this study.

\section{Acknowledgements}

The study was financially supported by the Tumour and Angiogenesis Research Group.

\section{References}

1 Koukourakis MI, Giatromanolaki A, Sivridis E, Pitiakoudis M, Gatter KC and Harris AL: Beclin-1 over-and underexpression in colorectal cancer: Distinct patterns relate to prognosis and tumour hypoxia. Br J Cancer 103: 1209-1214, 2010.

2 Lee YJ, Hah YJ, Kang YN, Kang KJ, Hwang JS, Chung WJ, Cho KB, Park KS, Kim ES, Seo HY, Kim MK, Park KG and Jang BK: The autophagy-related marker LC3 can predict prognosis in human hepatocellular carcinoma. PLoS One 8: e81540, 2013.

3 Fujii S, Mitsunaga S, Yamazaki M, Hasebe T, Ishii G, Kojima M, Kinoshita T, Ueno T, Esumi $\mathrm{H}$ and Ochiai A: Autophagy is activated in pancreatic cancer cells and correlates with poor patient outcome. Cancer Sci 99: 1813-1819, 2008.

4 Wu J, Dang Y, Su W, Liu C, Ma H, Shan Y, Pei Y, Wan B, Guo $\mathrm{J}$ and $\mathrm{Yu} \mathrm{L}$ : Molecular cloning and characterization of rat LC3A and LC3B-two novel markers of autophagosome. Biochem Biophys Res Commun 339: 437-442, 2006.
5 He H, Dang Y, Dai F, Guo Z, Wu J, She X, Pei Y, Chen Y, Ling W, Wu C, Zhao S, Liu JO and Yu L: Post-translational modifications of three members of the human MAP1LC3 family and detection of a novel type of modification for MAP1LC3B. J Biol Chem 278: 29278-29287, 2003.

6 Koukourakis MI, Kalamida D, Giatromanolaki A, Zois CE, Sivridis E, Pouliliou S, Mitrakas A, Gatter KC and Harris AL: Autophagosome proteins LC3A, LC3B and LC3C have distinct subcellular distribution kinetics and expression in cancer cell lines. PLoS One 10: e0137675, 2015.

7 Sivridis E, Giatromanolaki A, Liberis V and Koukourakis MI: Autophagy in endometrial carcinomas and prognostic relevance of 'stone-like' structures (SLS): What is destined for the atypical endometrial hyperplasia? Autophagy 7: 74-82, 2011.

8 Giatromanolaki A, Koukourakis MI, Harris AL, Polychronidis A, Gatter KC and Sivridis: Prognostic relevance of light chain 3 (LC3A) autophagy patterns in colorectal adenocarcinomas. J Clin Pathol 63: 867-872, 2010.

9 Sivridis E, Koukourakis MI, Zois CE, Ledaki I, Ferguson DJ, Harris AL, Gatter KC and Giatromanolaki A: LC3A-positive light microscopy detected patterns of autophagy and prognosis in operable breast carcinomas. Am J Pathol 176: 2477-2489, 2010.

10 Sivridis E, Giatromanolaki A, Zois C and Koukourakis MI: The 'stone-like' pattern of autophagy in human epithelial tumors and tumor-like lesions: An approach to the clinical outcome. Autophagy 6: 830-833, 2010.

11 Giatromanolaki A, Sivridis E, Mitrakas A, Kalamida D, Zois CE, Haider S, Piperidou C, Pappa A, Gatter KC, Harris AL and Koukourakis MI: Autophagy and lysosomal related protein expression patterns in human glioblastoma. Cancer Biol Ther 15: 1468-1478, 2014.

12 International Union Against Cancer: TNM Classification of Malignant Tumours. Sixth Edition. New York, NY: Wiley and Sons; 2002.

13 Klionsky DJ, Abdelmohsen K, Abe A, Abedin MJ, Abeliovich $\mathrm{H}$, Acevedo Arozena A et al: Guidelines for the use and interpretation of assays for monitoring autophagy (3rd edition). Autophagy 12: 1-222, 2016.

14 Guo JY and White E: Autophagy, metabolism, and cancer. Cold Spring Harb Symp Quant Biol pii: 030981, 2017.

15 Cao Y and Klionsky DJ: Physiological functions of ATG6/beclin1: A unique autophagy-related protein. Cell Res 17: 839-849, 2007.

16 Chen YB, Hou JH, Feng XY, Chen S, Zhou ZW, Zhang XS and Cai MY: Decreased expression of beclin-1 correlates with a metastatic phenotypic feature and adverse prognosis of gastric carcinomas. J Surg Oncol 105: 542-547, 2012.

17 Zhou WH, Tang F, Xu J, Wu X, Yang SB, Feng ZY, Ding YG, Wan XB, Guan Z, Li HG, Lin DJ, Shao CK and Liu Q: Low expression of beclin-1, associated with high BCL-xL, predicts a malignant phenotype and poor prognosis of gastric cancer. Autophagy 8: 389-400, 2012.

18 Geng QR, Xu DZ, He LJ, Lu JB, Zhou ZW, Zhan YQ and Lu Y: Beclin-1 expression is a significant predictor of survival in patients with lymph node-positive gastric cancer. PLoS One 7: e45968, 2017.

19 Yu M, Gou WF, Zhao S, Xiao LJ, Mao XY, Xing YN, Takahashi $\mathrm{H}$, Takano $\mathrm{Y}$ and Zheng HC: beclin-1 expression is an independent prognostic factor for gastric carcinomas. Tumour Biol 34: 1071-1083, 2013. 
20 Won KY, Kim GY, Lim SJ, Sung JY, Kim YW, Park YK, Lee J and Choi HS: Autophagy is related to the hedgehog signaling pathway in human gastric adenocarcinoma: prognostic significance of beclin-1 and GLI2 expression in human gastric adenocarcinoma. Pathol Res Pract 211: 308-315, 2015.

21 Qu B, Yao L, Ma HL, Chen HL, Zhang Z and Xie J: Prognostic significance of autophagy-related proteins expression in resected human gastric adenocarcinoma. J Huazhong Univ Sci Technolog Med Sci 37: 37-43, 2017.

22 Gill S, Shah A, Le N, Cook EF and Yoshida EM: Asian ethnicity-related differences in gastric cancer presentation and outcome among patients treated at a Canadian cancer center. J Clin Oncol 21: 2070-2076, 2003.

23 Theuer CP, Kurosaki T, Ziogas A, Butler J and Anton-Culver H: Asian patients with gastric carcinoma in the United States exhibit unique clinical features and superior overall and cancer specific survival rates. Cancer 89: 1883-1892, 2000.

24 Scarlatti F, Maffei R, Beau I, Codogno P and Ghidoni R: Role of non-canonical beclin-1-independent autophagy in cell death induced by resveratrol in human breast cancer cells. Cell Death Differ 15: 1318-1329, 2008.
$25 \mathrm{Wu}$ CA, Huang DY and Lin WW: Beclin-1-independent autophagy positively regulates internal ribosomal entry sitedependent translation of hypoxia-inducible factor $1 \alpha$ under nutrient deprivation. Oncotarget 5: 7525-7539, 2014.

26 Rohatgi RA and Shaw LM: An autophagy-independent function for beclin-1 in cancer. Mol Cell Oncol 3 pii: e1030539, 2016.

27 Liao W, Sun L, Wang C, Huang H, Liu J, Liao W and Shi M: LC3A-positive 'stone-like' structures predict an adverse prognosis of gastric cancer. Anat Rec 297: 653-662, 2014.

28 Yu S, Li G, Wang Z, Wang Z, Chen C, Cai S and He Y: Low expression of MAP1LC3B, associated with low Beclin-1, predicts lymph node metastasis and poor prognosis of gastric cancer. Tumour Biol 37: 15007-15017, 2016.

Received October 14, 2018

Revised October 23, 2018

Accepted October 26, 2018 